\title{
A Novel Trigger-Based Method for Hydrothermal Vents Prospecting using an Autonomous Underwater Robot
}

\author{
Gabriele Ferri ${ }^{1}$, Michael V. Jakuba ${ }^{2}$, Dana R. Yoerger ${ }^{3}$ \\ e-mail: g.ferri@sssup.it \\ tel.: +39050883396 , fax.: +39050883497 \\ 1 CRIM Laboratory \\ Scuola Superiore Sant'Anna \\ 2 Australian Center for Field Robotics \\ ${ }^{3}$ Deep Submergence Laboratory \\ University of Sydney \\ Woods Hole Oceanographic \\ Institution \\ Viale Rinaldo Piaggio 34, 56025 \\ Rose Street Bldg, J04 \\ Woods Hole, MA 02543, USA \\ Pontedera (Pisa), Italy \\ Sydney, NSW, 2006 \\ Australia
}

\begin{abstract}
In this paper we address the problem of localizing active hydrothermal vents on the seafloor using an Autonomous Underwater Vehicle (AUV). The plumes emitted by hydrothermal vents are the result of thermal and chemical inputs from submarine hot spring systems into the overlying ocean. The Woods Hole Oceanographic Institution's Autonomous Benthic Explorer (ABE) AUV has successfully localized previously undiscovered hydrothermal vent fields in several recent vent prospecting expeditions. These expeditions utilized the AUV for a three-stage, nested survey strategy approach (German et al., 2008). Each stage consists of a survey flown at successively deeper depths through easier to detect but spatially more constrained vent fluids. Ideally this sequence of surveys culminates in photographic evidence of the vent fields themselves. In this work we introduce a new adaptive strategy for an AUV's movement during the first, highest-altitude survey: the AUV initially moves along pre-designed tracklines but certain conditions can trigger an adaptive movement that is likely to acquire additional high value data for vent localization. The trigger threshold is changed during the mission, adapting the method to the different survey profiles the robot may find. The proposed algorithm is vetted on data from previous $\mathrm{ABE}$ missions and measures of efficiency presented.
\end{abstract}

Keywords: $A U V$, adaptive survey, hydrothermal vents prospecting, chemical plume tracing

\section{Introduction}

Since the first discovery of hydrothermal vents in 1979 (Corliss et al., 1979), these deep ocean phenomena have catalyzed an enormous interest in the scientific community for different reasons: the hydrothermal circulation of seawater through 
the oceanic crust influences many geological and oceanographic processes such as loss of heat from the earth, aging of the oceanic crust, geochemical cycling of the elements, biogeochemistry of deep ocean waters, and possibly general ocean circulation (Kadko et al., 1995). Also the biology of hydrothermal vents communities presents unique features (Fisher, 1995) and these biotas can be useful in understanding the origin of life on Earth (Zierenberg et al., 2000). On a series of recent expeditions we have shown that an AUV can aid the scientific characterization of seafloor venting in a way that is complementary to an approach reliant on ship borne assets alone (German et al., 2008). Providing scientific access to seafloor vent sites requires first localizing the vents themselves, the aspect of AUV-based hydrothermal exploration investigated here.

\subsection{Hydrothermal exploration}

A hydrothermal plume consists of hot, chemically altered seawater that is persistently discharged, or vented, as a turbulent buoyant plume (BP) that rises from the seafloor (Fig. 1). As it rises, the BP expands in diameter by incorporating surrounding seawater through the process of turbulent entrainment (Turner, 1986). Turbulent entrainment of denser seawater gradually reduces the buoyancy of the plume until neutral buoyancy is attained, at which point the remaining momentum causes the plume to overshoot and then sink back down until neutral buoyancy is again attained (McDuff, 1995). Eventually it forms a layer on the order of $100 \mathrm{~m}$ thick known as the non-buoyant plume (NBP) and spreads laterally. The NBP evolves under the combined effects of further turbulent mixing and advection driven by large-scale, tidally-forced, and self-induced currents (Helfrich and Speer, 1995).

The spatial scale of the NBP and BP are vastly different, a fact which has important implications for hydrothermal exploration. Whereas the NBP is detectable in situ with standard oceanographic instrumentation up to tens of kilometers from the source (Baker, 1995), BPs occupy relatively small spatial scales making them valuable indicators of nearby venting. Simulations in (Lavelle, 1997) show BPs attaining cross-stream widths of a few tens of meters and somewhat longer downstream tails of roughly $100 \mathrm{~m}$ in length before rising to the height corresponding to the bottom of the associated NBP. Thus if this buoyant stem can be found, then its associated vent is on the order of $100 \mathrm{~m}$ away. 
In practice, a kind of gradient search is executed in which lowered and towed instrumentation deployed from a surface ship is used to create profiles and vertical transects of the NBP in order to reveal high intensity regions of the NBP for additional survey (Baker, 1995). There is no fundamental limit to the resolution attainable this way and it has been historically adequate to localize venting; however, the process can be slow. Towed instrumentation on up to several kilometers of cable is difficult to maneuver, necessitating large excursions to execute turns. Lowered instrumentation requires hauling instruments up and down through the entire water column at each sampling location just to capture the deepest few hundred meters where NBPs reside. The speed with which such surveys can be completed is important in order to utilize ship time effectively.

On recent expeditions we have shown that AUVs can offer a capability that is complementary to lowered and towed assets (Yoerger et al., 2007; German et al., 2008). To date AUV endurance limits have required the use of ship borne assets to constrain survey areas to a few square kilometers; however, at this scale an AUV's maneuverability permits the rapid creation of high resolution maps of the NBP while freeing the support ship to conduct other activities. These maps are interpreted in terms of likely vent locations, after which we have employed a series of targeted nested surveys at successively lower heights above the seafloor to home in on vent sites. The procedure is described in detail in (German et al., 2008) and Fig. 3 shows an example set of tracklines. This nested strategy capitalizes on our AUV's multimodal imaging capabilities to generate bathymetric maps and photomosaics around the vent sites themselves. These maps provide important geological context and a preliminary assessment of available habitat and macrofaunal assemblages to guide subsequent scientific investigation (German et al., 2008).

This nested strategy for homing in on vent sites has been particularly successful when BPs can be directly identified in data from a NBP survey. AUVs have limited energy capacity, so that while decreasing trackline spacing makes direct BP interception more likely, it also reduces the area that can be covered by a survey, and a high resolution survey with tightly spaced tracklines is worthless if conducted in the wrong place. For completely preplanned surveys of the type reported in (German, 2008), this trade-off places a substantial burden on the scientific party to decide when to complete towed and lowered survey efforts in 
favor of deploying the AUV, and then how to arrange the tracklines of its mission. Improving the coverage attainable from a single AUV dive without compromising the ability to directly intercept BPs would require a less precisely defined survey area. We present simulation studies using real data collected by an AUV surveying in NBPs that suggest this is possible by capitalizing on an AUV's inherent capacity to react to sensed data in real time.

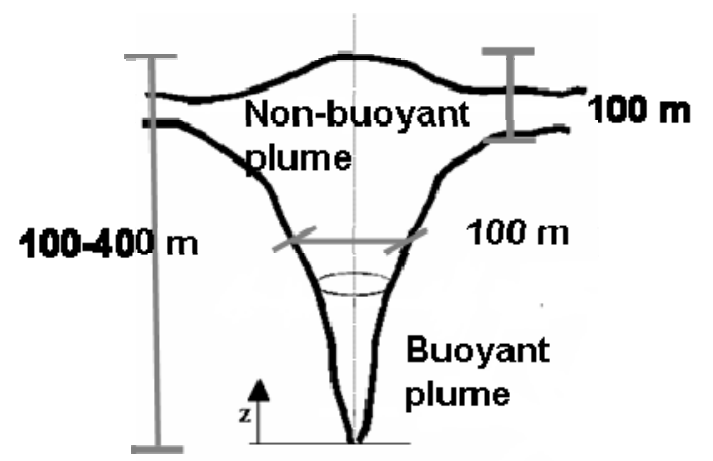

Fig. 1 Hydrothermal plume schematic with characteristic dimensions. The plume's rise height can reach 100-400 $\mathrm{m}$ in typical hydrographic settings. In a time-averaged sense a buoyant plume expands laterally, during its rise, from a diameter of few $\mathrm{cm}$ to a diameter on the order of $100 \mathrm{~m}$. The non-buoyant plume presents a vertical thickness on the order of $100 \mathrm{~m}$.

\subsection{Autonomous Benthic Explorer (ABE) and three-stage strategy}

The specific AUV considered here is the Woods Hole Oceanographic Institution's Autonomous Benthic Explorer (ABE) (Yoerger et al. 1991) AUV (Fig. 2). Large amount of data from historic missions are available so we can assess the method.

The Woods Hole Oceanographic Institution's Autonomous Benthic Explorer (ABE) (Yoerger et al. 1991) AUV (see Fig. 2) has been successfully used in several hydrothermal vents prospecting missions, (German et al., 2005; Jakuba et al., 2005; Yoerger et al., 2007). ABE is highly maneuverable and it can achieve a precision in self-localization of the order of meters using external acoustical beacons (Yoerger et al., 2007). Its cruise speed is about $0.6 \mathrm{~m} / \mathrm{s}$ and for its payload it can carry different sensors for detecting anomalies in the water caused by venting activities. All these features allow ABE to build a map with higher spatial resolution (improved maneuverability) and temporally closer to a synoptic view (faster speed) with respect to those achievable using towed assets. Its autonomy during the missions, peculiar of AUVs, allows the vessel crew to do 
other work during the surveys. Finally, the computational capabilities available aboard on ABE enable data-driven strategies. This could increase the efficiency of single surveys in acquiring information about hydrothermal activity.

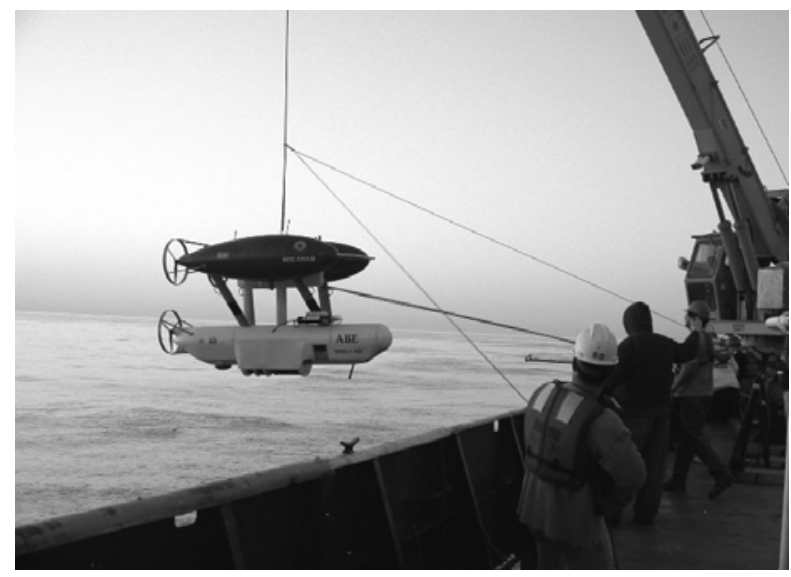

Fig. 2 Deployment of the Autonomous Benthic Explorer (ABE) vehicle.

Recently, a three-stage, nested approach to vents localization has been introduced with success (Langmuir et al., 2004) consisting in surveys at different depths to finally photograph the venting structures flying very close to the seafloor (German et al., 2008). ABE performs surveys at three successively lower altitudes. It covers pre-designed tracklines that become finer spaced and cover a smaller area as the survey heights decrease (see Fig. 3). At the end of each survey, the data collected by the robot are scrutinized by the scientific party on the ship, together with any available ancillary data and a new dive is planned. Ideally this sequence of surveys culminates in photographic evidence of the vent fields themselves.

In this paper, we propose a new movement strategy called triggered spirals prospecting (TSP) exploiting ABE's computational capabilities and particularly suited to surveys at higher altitude (200-400 m) (Phase-1), that is the most difficult for several reasons:

- high altitude implies weaker signals revealing a plume and that the measurement of water current is difficult or misleading;

- the wide-spaced tracklines of Phase-1 cause difficulties in covering the search area.

It aims at triggering additional tightly spaced survey tracklines at locations likely to contain active venting areas. These additional tracklines increase the 
probability of direct contact with buoyant plumes thus helping to constrain vent field location. Our strategy avoids relying on often misleading and difficult to analyze information carried by the water current direction and intensity.

In the following section we will describe the difficulties related to Phase-1 and to the use of data-driven strategies. Next, TSP is described. In Section 3, results using data coming from ABE's previous surveys are presented and discussed. Finally conclusions and recommendations for future research are presented.

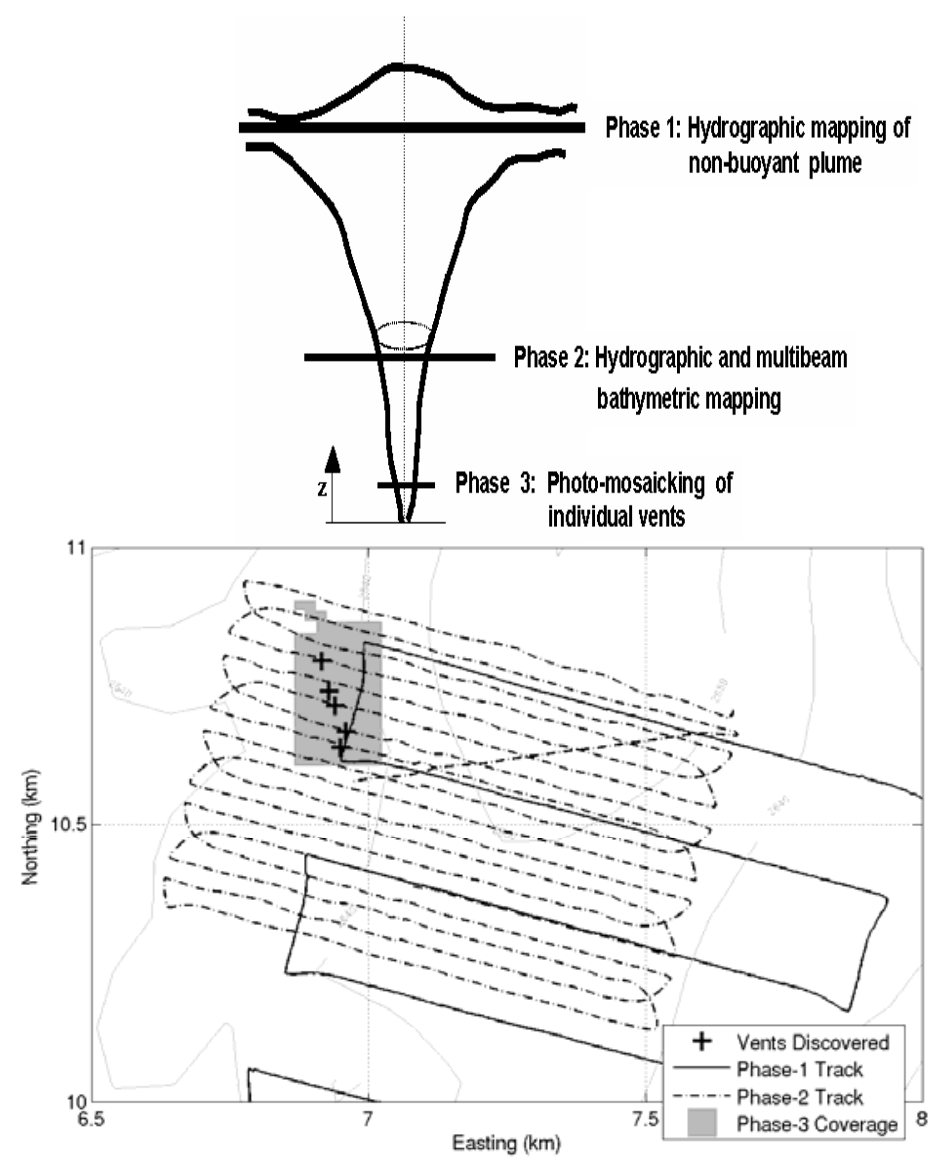

Fig. 3 (Top) Schematic of a hydrothermal plume with the different altitudes for the three-phase surveys. (Bottom) Threestage surveys tracklines covered by ABE at "Kilo Moana" vent field on Eastern Lau Spreading Center $\left(20^{\circ} 3^{\prime} \mathrm{S}, 176^{\circ} 12^{\prime}\right.$ W) (Mid-Pacific Ridge). The Phase-1 survey continues several $\mathrm{km}$ to the south of the region plotted.

\subsection{Phase-1 and data-driven strategies issues}

The three-stage, nested approach has proved to work with success in different expeditions to localize active hydrothermal sites (Langmuir et al., 2004; German et al., 2005; German et al., 2008). An automation or a support to human operator in analyzing the acquired information would improve reliability and efficiency in 
designing next surveys. For example, the ratio of time associated with intersurvey recovery, maintenance and deployment related to the time spent collecting data is roughly $2: 1$.

Most importantly, the ability of ABE to move autonomously driven by the collected data can increase the possibility to explore interesting areas thereby enhancing the useful amount of information available for the design of successive dives and reducing the number of dives (time) necessary to localize the buoyant plumes.

Autonomous decisions made by ABE in Phase-1 based on collected data have the greatest potential for improving the efficiency of the three-phase strategy.

We focus in this paper exclusively on this phase. The altitude of the survey implies that the measured anomalies are lower in magnitude but of increased spatial extent relative to those encountered to lower heights. Turbulence makes the detectable anomalies patchy (Lupton, 1995). These features make it difficult, at the height of Phase-1 surveys, to correlate measured anomalies with likely venting sources positions on the seafloor. Direct interceptions with rising stems (typical diameters are $\mathrm{O}(100 \mathrm{~m})$ ) are rare given the large survey area and wide trackline spacing (we have employed trackline spaces during Phase-1 surveys between $200 \mathrm{~m}$ and $1000 \mathrm{~m}$ ). The wide spacing is needed to cover the large areas typical of Phase-1 (20-25 km²) before the batteries are depleted (up to $30 \mathrm{hrs}$ of battery life). To further complicate the situation, ambient crossflows tend to bend the buoyant plumes and also tidal oscillatory currents can produce areas with strong anomalies not connected with nearby nascent plumes (Veirs, 2003). Measurements at the Endeavour Segment of the Juan de Fuca Ridge (Thompson et al., 2003) reveal tidally-forced oscillatory currents of up to $10 \mathrm{~cm} / \mathrm{s}$ with superimposed steady current of $5 \mathrm{~cm} / \mathrm{s}$. The maximum crossflow magnitude observed by ABE from on-board ADCP measurements as of 2006 was $16 \mathrm{~cm} / \mathrm{s}$ at a site on the Southern Mid-Atlantic Ridge (SMAR). Tidal currents have a dominant $12 \mathrm{hrs}$ period, for this reason the plume can change significantly on scales of $\mathrm{O}(10 \mathrm{~h})$, the same timescale of one of ABE's dives (Jakuba et al., 2005). Finally, the flow measurements are not always accurate due to the loss of bottom track.

In these conditions, the use of gradient ascent-based (Burian et al., 1996) and bio-mimetic methods ( $\mathrm{Li}$ et al., 2006) appears to be problematic. The absence of a 
well defined gradient and the fact that high intensity anomalies caused by tidal currents may be found far away from the source imply gradient ascent-based methods are liable to wander or to get "stuck" near local maxima. Bio-mimetic schemes usually rely on measurement of wind/crossflow to trace the plume up to the source (Li et al., 2006). In Phase-1 surveys, the oscillatory tidal components and the difficulties in having a precise measure of the flow can confound them. We emphasize that given the intrinsic multiple-source nature of the problem, the use of a purely reactive/behaviour based algorithm does not guarantee the needed exploration of the survey area. Most reactive or behavior-based plume tracing algorithms implicitly assume that only a single source is present in the environment and they offer no guidance on how to continue searching for other sources once a source has been found, or how to avoid re-finding the same source. Some work has been done to move ABE autonomously. An algorithm, planning autonomously the robot's path based on acquired data, has been proposed and tested (Jakuba et al., 2005; Yoerger et al., 2007) for hydrothermal vents prospecting: at the end of the pre-planned survey new tracklines are added starting on interesting locations chosen after the tracers anomalies are propagated to the seafloor using a plume model and crossflow measurements. This method was applied in Phase-3 dives. Even if it is quite conservative it improved the efficiency of Phase-3 dive: additional tracklines comprised only 5\% of total mission time and resulted in a $36 \%$ of high-value data collected (Yoerger et al., 2007). This shows how autonomously data-driven strategies can improve the efficiency of dives. However, the main obstacle to apply this method to Phase-1 surveys is that it chooses the locations to investigate further once all the pre-planned tracklines have been covered. The large spatial extension of Phase-1 would make it not feasible for $\mathrm{ABE}$ to come back on the chosen locations.

The two paradigms for searching for a chemical source (data-driven approach and pre-designed tracklines) have therefore different features: while data-driven strategies are able to increase the efficiency of missions, they do not assure a uniform covering of the whole search area guaranteed by fixed pre-planned trajectories. Preplanned tracklines guarantee coverage but at a fixed resolution. When much of a dataset can consist of relatively uninteresting background as is the case of Phase-1 surveys, preplanned surveys are likely to return with insufficiently high resolution data in the few areas of interest. Data-adaptive 
surveys can alleviate this problem to an extent but an accurate coverage of the area remains important.

The TSP approach aims at mixing the achieving of the guaranteed coverage of pre-designed tracklines with the efficiency of data-driven strategies without considering the often misleading information carried by the water current.

\section{TSP algorithm}

The objective of TSP is to enable ABE to further explore areas that are likely to contain nascent hydrothermal plumes while nominally executing a pre-planned path. Since these additional tracklines are likely to result in direct contact with BP water, the additional collected information is likely to be invaluable for delimiting regions likely to contain vents. In the event that the data do not suggest a nearby BP the additional data will likely disambiguate otherwise uncertain locations.

The large spatial extent of Phase-1 surveys imposes the constraint that the robot has to choose the locations to explore when it is not too far from them going on its pre-planned path. In this way, it can come back to the chosen locations without wasting too much mission time. This constrains TSP to trigger the movements based only on past acquired information: we cannot complete the survey and then decide which locations to investigate (this strategy is pursued in the method proposed in (Jakuba et al., 2005; Yoerger et al., 2007) where the smaller areas covered in Phase-3 allow to pursue this approach).

In our approach, $\mathrm{ABE}$ moves along pre-designed tracklines sampling the seawater. When an area containing clues of the presence of young hydrothermal waters (anomalous values of some hydrothermal activity tracers) is found, a spiral movement is triggered to acquire more information about that area. The number of spirals is constrained by ABE's energy capacity so only the highest anomalies locations have to be investigated. The spiral arms length, shape and orientation are pre-designed.

The problems to solve in this strategy are:

- determine which tracers to consider as clues for nearby nascent hydrothermal plumes in order to realize when the robot is crossing areas containing potentially useful information; 
- develop a methodology to elaborate the data to compute anomalies in realtime;

- a method aiming at maximizing the likelihood of considering as possible triggers during the surveys only the locations with the highest intensity clues of the whole survey.

\subsection{Tracers for nearby nascent hydrothermal plumes}

Different tracers can be used to reveal the presence of hydrothermal plumes (Baker et al., 1995). In our case, we need tracers that present two features: they have to be reliable clues of nearby nascent hydrothermal plumes (not exclusively buoyant) and the anomalies with respect to background waters have to be computable in real-time.

From the different tracers collected by ABE during recent missions (Jakuba, 2007) the most suited tracer presenting these two features is the Eh anomaly in the water column.

Reduction-oxidation potential (Eh) is a measure of the capability of chemical species in water to oxidize (positive Eh) or to reduce (negative Eh). The Eh potential has been measured by $\mathrm{ABE}$ using a sensor developed by Dr. Ko-ichi Nakamura ${ }^{1}$ and has been shown to be a good tracer to detect nascent hydrothermal fluid (Nakamura et al., 2000). The nascent rising plume contains reducing chemicals that are rapidly oxidized as the plume matures (Nakamura et al., 2000). A low value of Eh, therefore, is a distinctive feature of young hydrothermal plumes. The Eh sensor has a complicated response characterized by a fast onset and a slow recovery time. Ongoing work by Dr. Nakamura suggests that the magnitude of $\mathrm{d} / \mathrm{dt}(\mathrm{Eh})$ is strongly correlated with the chemicals typically present in young hydrothermal plumes (Nakamura, personal communication 2006): steeply decreasing Eh signals appear to be a reliable sign of the interception of the younger part of hydrothermal plumes (not exclusively buoyant plumes). The instrument presents a relatively flat response in unaltered water allowing to compute a background in real-time..

\footnotetext{
1 National Institute of Advanced Industrial Science and Technology (AIST), Institute for Marine Resources and Environment, Seafloor Environment and Resources Research Group 1, Tsukuba, Ibaraki, 305- 8567 Japan
} 
For these reasons we will consider Eh anomalies as a trigger signal for spiral movements. In the next two sections we show how anomalies are quantified and declared.

\subsection{Eh anomaly measure}

Quantifying anomalies in Eh is not a trivial task. In our experience, they are characterized by anomalously low $\mathrm{d} / \mathrm{dt}(\mathrm{Eh})$ and by a relatively significant variance in $\mathrm{d} / \mathrm{dt}(\mathrm{Eh})$ values in a small number of samples (this is evident from Fig. 4). The first step to declare Eh anomalies is to find a way of quantifying what our experience suggests. We consider the median absolute deviation (MAD) as a measure of variability of the $\mathrm{d} / \mathrm{dt}(\mathrm{Eh})$ signal in an interval. MAD is defined as the median absolute deviation from the median,

$M A D(x)=\operatorname{med}|x-\operatorname{med}(x)|$

where $x$ is a data vector and med is the median operator. MAD is a measure of the variability of data in an interval robust to outliers eventually present in the data set (Huber, 1981).

To process Eh data during the survey we compute MADs on a fixed temporal window as the data are being collected by ABE. To do this, the Eh signal acquired from the sensor is first low pass filtered and then a numerical derivative is computed. Then, every $N$ samples, a MAD is computed on $\mathrm{d} / \mathrm{dt}(\mathrm{Eh})$ signal ( $N$ is chosen to create a new window every $50 \mathrm{~s}$ ). As a result, we produce for every temporal window $i$ one MAD, denoted $M A D_{i}$.

We have now a number $\left(M A D_{i}\right)$ that represents a measurement of the variability of $\mathrm{d} / \mathrm{dt}(\mathrm{Eh})$ in each $N$-sample temporal window. A high $M A D_{i}$ means the temporal window $i$ exhibits strong variations in $\mathrm{d} / \mathrm{dt}(\mathrm{Eh})$ signal showing that the robot is crossing waters likely altered by nascent hydrothermal waters.

A method to declare a $M A D_{i}$ as an anomaly has to be specified.

\subsubsection{Background creation and anomaly detection}

We approach the problem of anomalies declaration as a problem of outliers detection on computed $M A D_{i}$. The idea is to consider as outliers $M A D_{i}$ whose distance from the median of a reference vector of data is greater than a fixed threshold. The reference vector has to contain $M A D_{i}$ computed in an area where 
the water is not altered by hydrothermal vents. We considered a reference vector fixed in time during the survey because we assume no valuable drifts in Eh signal measurements: it is considered a background fixed in time with respect to which anomalously high $M A D_{i}$ can be detected. On the computed $M A D_{i}$ we calculate the median $\left(M E D_{B}\right)$ and the $\operatorname{MAD}\left(M A D_{B}\right)$, that is $M E D_{B}=\operatorname{med}\left(M A D_{i}\right)$ and $M A D_{B}=$ $\operatorname{MAD}\left(M A D_{i}\right)$ in both cases for all $i$ in the descent. During the survey, a new computed $M A D_{i}$ is considered as an outlier detection if:

$\left(M A D_{i}-M E D_{B}\right) \geq c \cdot 1.4286 \cdot M A D_{B}$

where $c=9$ is a constant derived from analysis of previous and 1.4286 is a normalization used to make the MAD an unbiased estimate of the standard deviation for Gaussian data (Huber, 1981). The idea behind using data-dependent threshold to discriminate an outlier is to first estimate the range of variation of the nominal data sequence and then assess each new element with respect to the median value of the nominal data sequence (considered as representative of the center of the sequence) and this estimated range of variation. To create the threshold we chose the MAD because it is a good measure of statistical dispersion and is more resilient to eventual outliers contamination than the usual standard deviation estimate (Menold et al., 1999). (2) allows us to compute the outliers in real-time and is similar to the method used in (Menold et al., 1999) for online outlier removal in data sequence. However, while in (Menold et al., 1999) the discriminating threshold is computed on a moving data window to detect outliers from data sequences, in our case is computed using the data of the background built during the robot's descent (during the survey we search for $M A D_{i}$ that are outliers with respect to the $M A D_{i}$ composing the background).

In our trials, we considered $N_{B}$ (number of $M A D_{i}$ used for the background creation) in order to compute the background on a temporal window of 50 minutes: a trade-off between assuring a less variable $M A D_{B}$ (wider windows) and avoiding the inclusion in the computation of altered areas potentially encountered towards the end of the descent. Finally, we exclude from anomalies declaration $M A D_{i}$ originated from windows with always positive $\mathrm{d} / \mathrm{dt}(\mathrm{Eh})$ signal (these windows derive from periods of slow recovery of Eh sensor with high variability of the signal). In Fig. 4 the process of $M A D_{i}$ creation from Eh measurements is shown. 
The $M A D_{i}$ declared as detections are related to periods (and areas) of the survey that are characterized by an increased variability of $\mathrm{d} / \mathrm{dt}(\mathrm{Eh})$. Methods to choose areas with the highest anomalies of the survey (highest values of $M A D_{i}$ ) have to be introduced. To decide which computed anomalies are worth triggering a spiral we adopt two methods: the first one is to group detections and the second one consists in lowering/raising the probability of triggering based on the data acquired so far (see Sec. 2.4. Trigger threshold). 

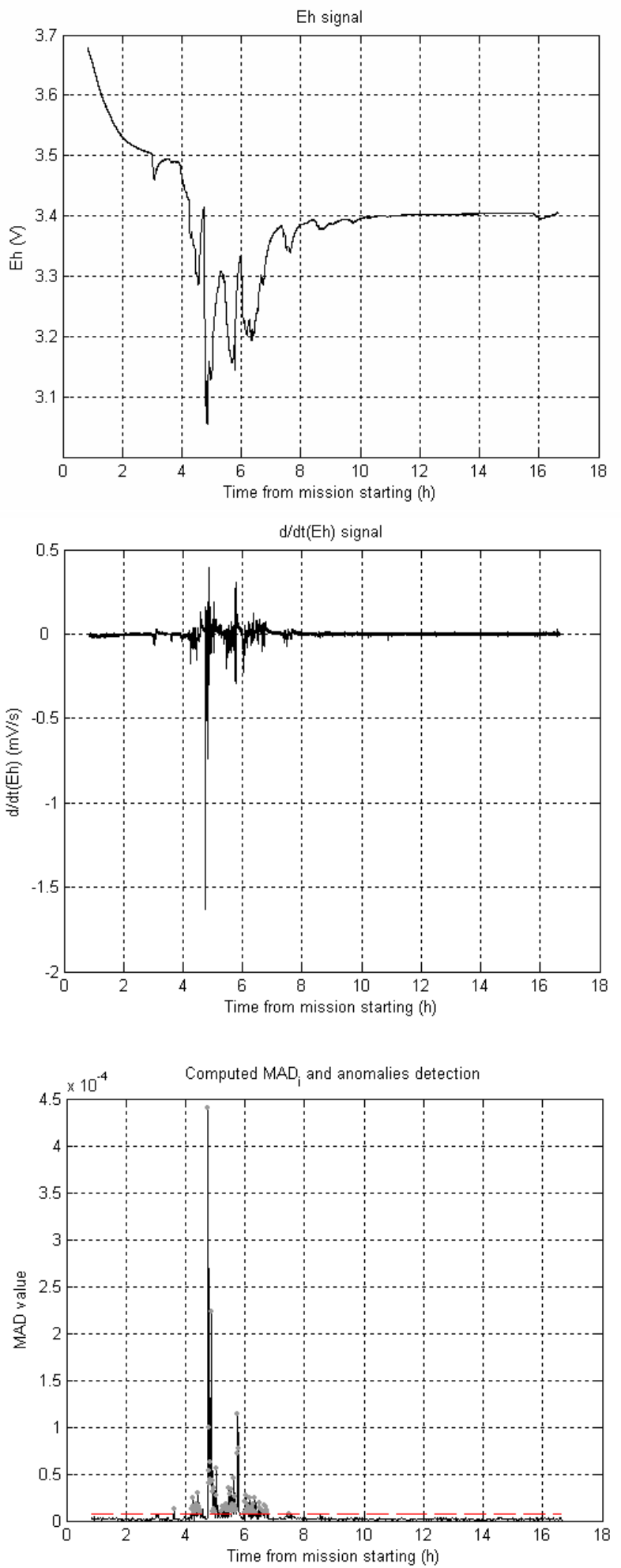

Fig. 4 Process of $M A D_{i}$ creation starting from Eh measurements coming from a Phase-1 at "Kilo Moana" vent field on Eastern Lau Spreading Center $\left(20^{\circ} 3^{\prime} \mathrm{S}, 176^{\circ} 12^{\prime} \mathrm{W}\right)$ (Mid-Pacific Ridge) are reported. At the top Eh signal and the filtered and derivated signal are reported. Finally, at the bottom, the computed $M A D_{i}$ are shown with grey dots marking the detected anomalies. The dashed line represents the threshold above which a $M A D_{i}$ is considered a detection. From the figure it is clearly visible how high $M A D_{i}$ are correlated with portions of $\mathrm{d} / \mathrm{dt}(\mathrm{Eh})$ signal characterized by high variability. 


\subsection{Patches and clusters creation}

The computed detections are grouped using a two-layered procedure: first they are aggregated into groups of detections (patches), and then the patches are grouped into clusters. The best patch (we have still to define what "best" patch means) of one cluster triggers a spiral movement. The creation of patches simplifies the description of widespread detections: patches carry information about groups of nearby detections. The clusters are a method for considering the information carried by nearby patches while choosing where to trigger: this helps in selecting the most interesting locations allowing at the same time to limit trigger movements in near locations.

Patches creation: a patch is started once a detection is encountered. Next $M A D_{i}$ declared as detections are assigned to the patch until the patch is finished. The patch is considered finished when there are no detections in consecutive $N_{\text {patch }}$ $M A D_{i}$ (see Fig. 5). $N_{\text {patch }}$ is chosen equal to 6 such that a time of about 5 minutes without detections cause the end of the patch (a length of the order of magnitude of a BP without a detection ends a patch). The patches are stored in memory, each one with its centroid chosen as the location of the highest $M A D_{i}$ belonging to the patch (the highest $M A D_{i}$ is called the patch value $\left.\left(V_{\text {patch }}\right)\right)$. The location of a $M A D_{i}$ is chosen as the position of the middle $\mathrm{d} / \mathrm{dt}(\mathrm{Eh})$ sample in the $N$-long data vector from which the $M A D_{i}$ is computed.

When a patch is closed, the value of the patch is compared with a trigger threshold $\left(\eta_{\text {trigger }}\right)$ : if it is greater, that is, if

$V_{\text {patch }} \geq \eta_{\text {trigger }}$

a cluster is started. The trigger threshold $\eta_{\text {trigger }}$ is the threshold that discriminates if a patch of anomalies is able to trigger a spiral movement. It is adaptively chosen to limit the number of spirals and to modify the trigger threshold on the basis of the data acquired during the survey. This is described in Sec. 2.4.

Clusters creation: when the condition

(3) is

verified for one patch, a cluster is started and the patch is added to the new cluster. Next created patches for which

(3) is true are 
added to the current cluster. The cluster is closed after a distance of $D_{\text {cluster }}$ meters from the centroid of the first patch of the cluster has been covered; if one patch is being created after $D_{\text {cluster }}$ meters the cluster is closed at the end of that patch (see Fig. 5). The $D_{\text {cluster }}$ value is a tunable parameter and represents how near we permit different spirals can be triggered. In our trials we chose a $D_{\text {cluster }}$ value constituting about the $2.5 \%$ of the total length of the tracklines covered by ABE. Once a cluster is closed, the patch in the cluster with the highest $V_{\text {patch }}$ (we consider this as the merit value for patches) is chosen and a spiral movement starting from the centroid of that patch is triggered.

The second method aiming at selecting only the highest $V_{\text {patch }}$ patches relies on how the trigger threshold is dynamically modified during the survey.

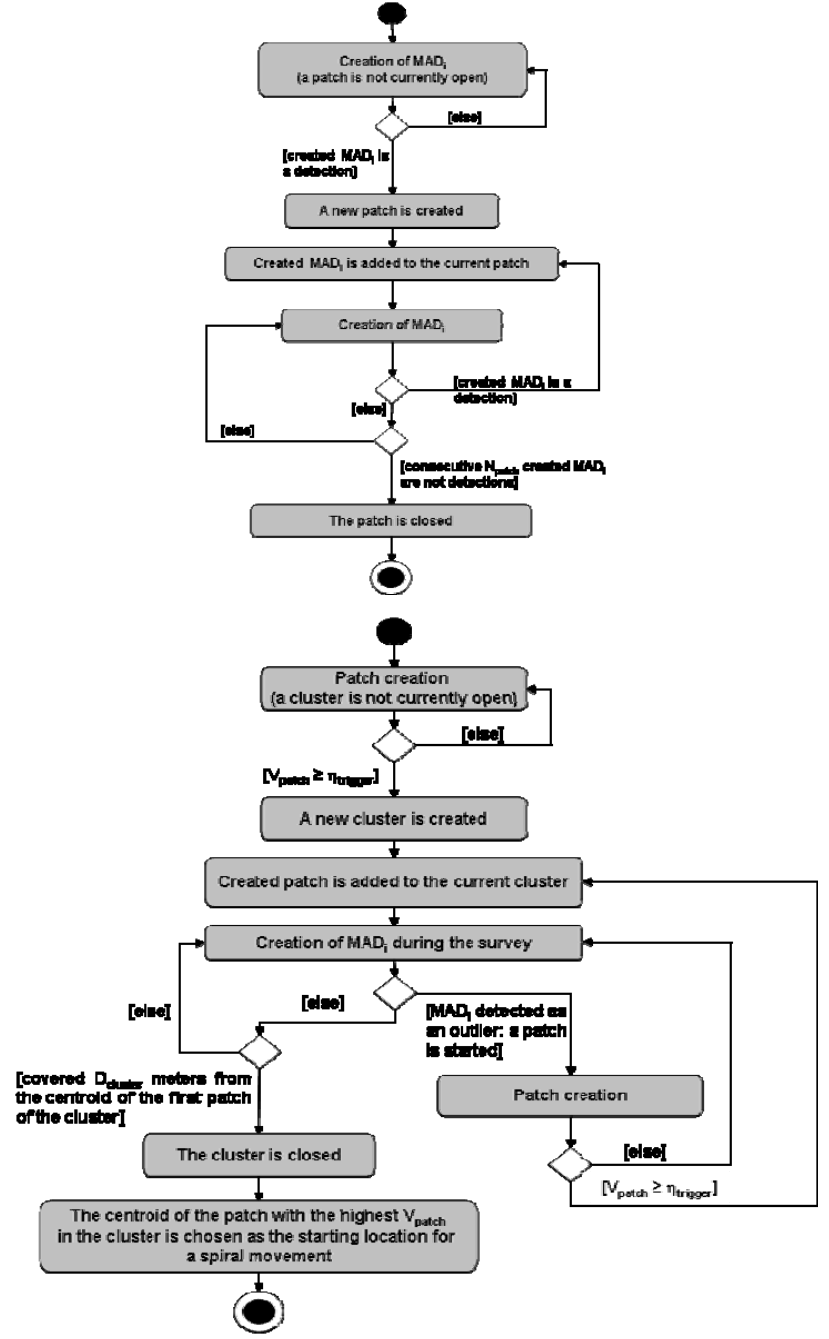

Fig. 5 (Top) Activity diagram of the patch creation and (bottom) activity diagram of the cluster creation. 


\subsection{Trigger threshold}

Phase-1 dives present different $\mathrm{d} / \mathrm{dt}(\mathrm{Eh})$ profiles: some present many areas with high intensity perturbations, others present low intensity anomalies. A static trigger threshold is not therefore the best solution: a threshold low with respect the values of $M A D_{i}$ created during a survey can cause too many spirals thereby consuming the batteries before something interesting is encountered; while a too high threshold can rarely trigger losing possible interesting areas.

We approach this problem using an adaptation mechanism for the threshold. At the beginning of the survey $\eta_{\text {trigger }}$ is fixed to the value $\eta_{\text {trigger } B}$. The unit of measure of $\eta_{\text {trigger }}$ is the normalized $M A D_{B}$. This avoids the difficult problem of providing an absolute value for the threshold. We use the value $\eta_{\text {trigger } B}=180$ based on our experience with Phase-1 collected data. The adapting mechanism depends on the approximate number of spirals we would like ABE to execute $(S S)$ and on how the survey is progressing. The number of suggested spirals is a design parameter and is an indication of how many spirals $\mathrm{ABE}$ is able to start. This is an important element of survey design. We want to be sure ABE has enough energy to cover the whole survey. However, it is not a constraining parameter in the sense that TSP allows that more or less spirals than $S S$ can be started even if it can be considered as a maximum number of allowed spirals. In all our trials we considered a value for $S S$ equal to 5 (see Sec.3 for further details on the choice of this parameter).

The adaptation works as follows: if the percentage of triggered spirals with respect to the suggested ones is lower than the percentage of covered tracklines then the threshold is lowered, otherwise it is increased. The threshold modifications depend on so far created $V_{\text {patch }}$ to modify the threshold in a way suitable to the conditions encountered during the actual dive. At the beginning of the survey, the threshold is slightly modified. As the robot covers a higher percentage of the scheduled tracklines the threshold is more liable to changes considering the increased knowledge about the area of the survey acquired by ABE. The algorithm for adapting the threshold is run whenever a new patch is closed. The adaptation algorithm is shown in Table 1 . 
Table 1. Algorithm for adaptive threshold.

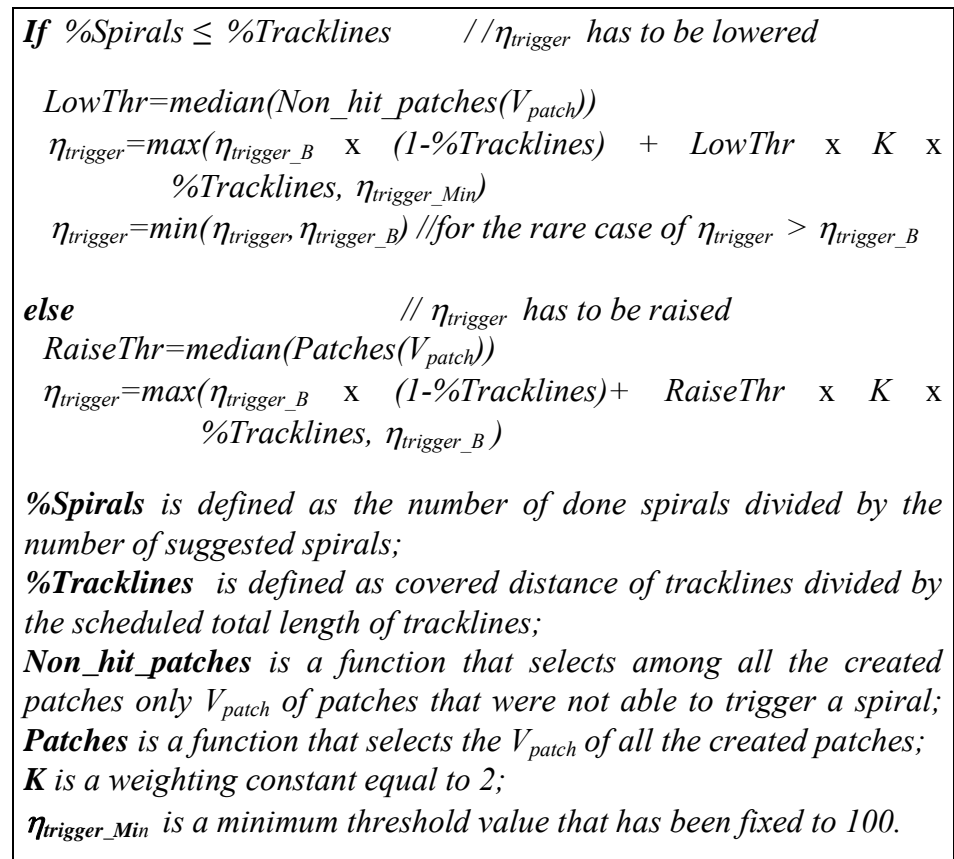

\subsection{Spiral movement}

One spiral movement is started whenever a cluster is closed. ABE comes back and reaches the centroid of the patch with the highest $V_{\text {patch }}$ in the cluster and starts a spiral. The spiral arms are predefined in extent, orientation and spacing (see Fig. 6). The basic spiral length $D$ is chosen in function of the trackline spacing: a wider spacing involves longer arms to allow ABE exploring the area between two tracklines. The choice of a spiral movement is not critical for the algorithm, however it is an effective way of exploring an area and can be used in the future (see Sec. 4) to propagate successive spirals like in the method proposed in (Ferri et al., 2009).

A state diagram of TSP algorithm is shown in Fig. 7.

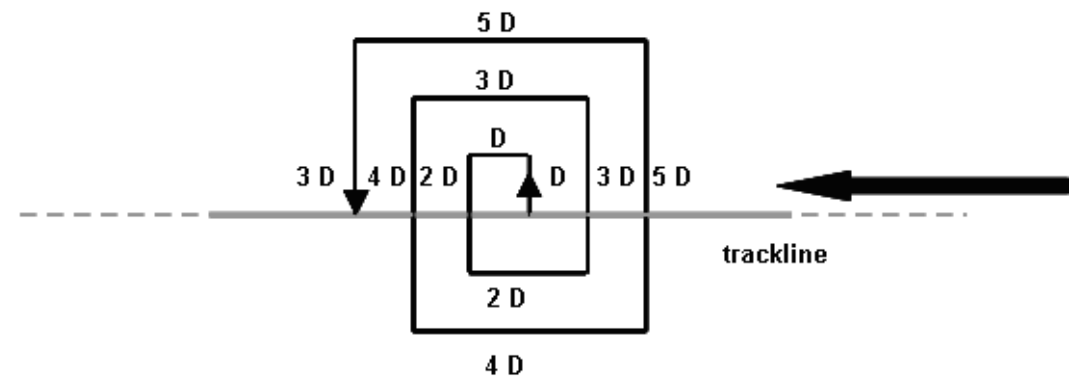

Fig. 6 Sketch of the pre-designed spiral movement. The only parameter changing the spiral is the basic spiral length D. The black arrow shows the direction of ABE on the trackline. 


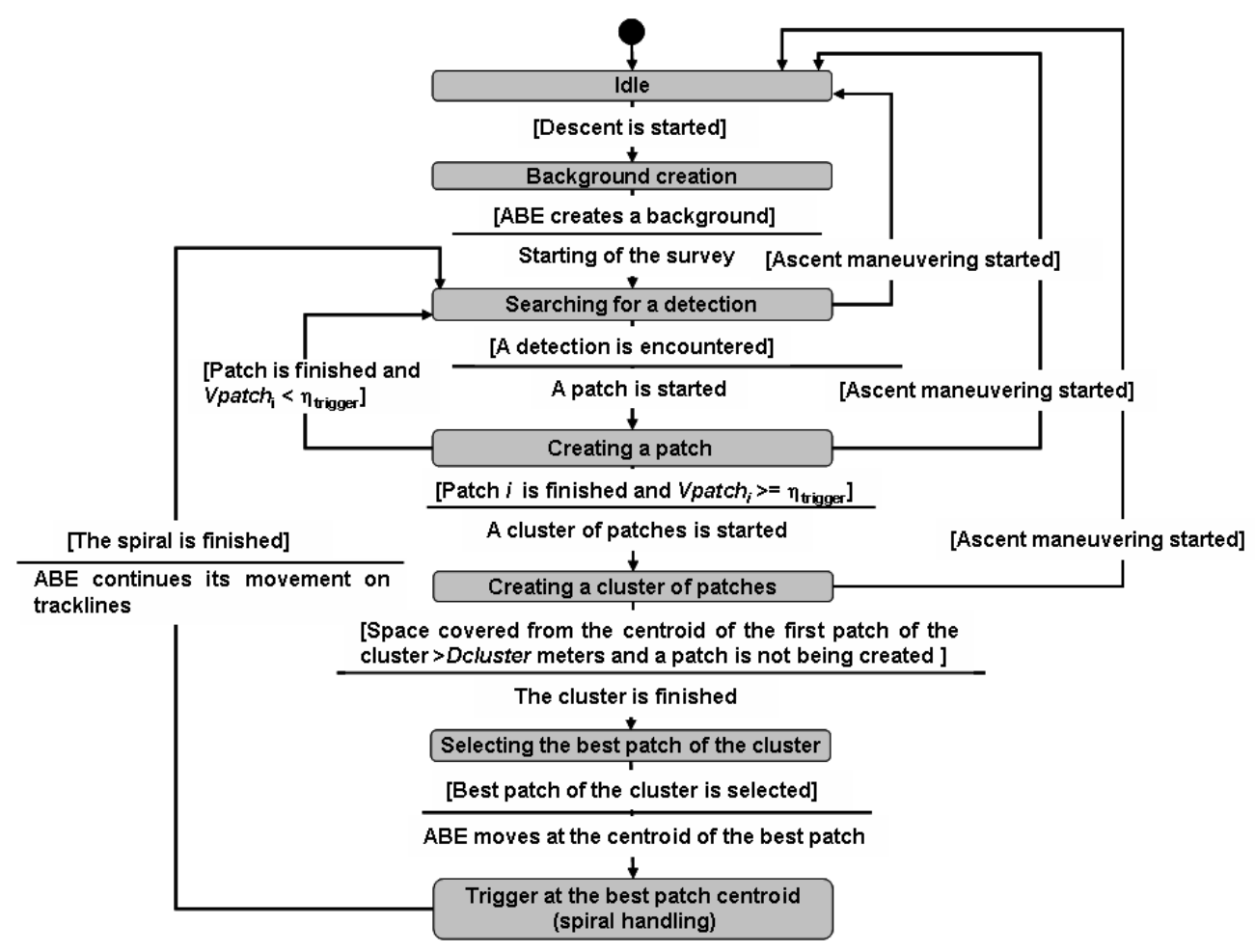

Fig. 7 State diagram of TSP algorithm.

\section{Results using TSP with data collected by ABE in previous surveys}

The proposed algorithm was tested using data collected by $\mathrm{ABE}$ in previous Phase-1 surveys. It was tested on 7 datasets of different dives. The values of the used parameters have been presented in previous sections. The only remaining parameter to be specified is the basic spiral length: in all the trials except one $D=55 \mathrm{~m}$. In one trial, given the wide spaced tracklines, we considered $D=150 \mathrm{~m}$. As shown in Fig. 6, the length of a spiral is $33 D(1815 \mathrm{~m}$ for $D=55$ and $4950 \mathrm{~m}$ for $D=150$ ). Considering the robot's speed of $0.6 \mathrm{~m} / \mathrm{s}, 30 \mathrm{hrs}$ battery life and a $10 \%$ caution factor, $\mathrm{ABE}$ can traverse roughly $50 \mathrm{~km}$ per deployment, thus one spiral costs between $4 \%$ and $10 \%$ of the entire mission. The suggested spirals parameter $(S S)$ was set to 5 because, including also the space covered by ABE to come back to a spiral starting location once a cluster is closed, 5 spirals compose from about $25 \%(D=55)$ to more than $50 \%(D=155)$ of the mission greatly 
reducing the amount of area that can be covered. From a temporal point of view, it takes about 50 minutes or $2.3 \mathrm{hrs}$ to the robot to cover a small or large spiral.

The algorithm was implemented by use of Matlab ${ }^{\circledR}$, from The MathWorks, Inc. The program acquires raw Eh signal recorded by $\mathrm{ABE}$ during the mission, the positions of the robot and computes the trajectory produced by TSP. The Eh data are sequentially fed into the program as they would be collected by the AUV; every 50 acquired samples, a new $M A D_{i}$ is generated. On $\mathrm{ABE}$ robot the Eh data are read from Dr. Nakamura's instrument with $1 \mathrm{~Hz}$ frequency. The execution of TSP would be therefore a periodic task with $1 \mathrm{~Hz}$ frequency. For what concerns the computational capability, ABE's main control board during the time frame in question was a MicroSys SBC1586-1-ET, with a $266 \mathrm{MHz}$ Low-Power Pentium CPU and $256 \mathrm{MB}$ of RAM memory. The ABE code used only a few percent of the CPU, nearly all the time the code was waiting for serial transactions to complete. Recently, ABE's main control board was upgraded to a Cool Roadrunner. With the new Cool Roadrunner and the same code, $<1 \%$ of the CPU time is used. Considering the operations performed by TSP and its frequency of execution, the computational power available on $\mathrm{ABE}$ allows an easy integration of the algorithm in the navigation and control system. The known locations of the vents are used to assess the performance of TSP algorithm in terms of the positions where the spirals are triggered.

We discuss in detail the results for three datasets showing different degrees of difficulty: the first dataset is from a Phase-1 survey at "Kilo Moana" site on Eastern Lau Spreading Center (ELSC) (20 $\left.3^{\circ} \mathrm{S}, 176^{\circ} 12^{\prime} \mathrm{W}\right)$ (Mid-Pacific Ridge), the second one is from a site at Southern Mid-Atlantic Ridge (SMAR) (4 ${ }^{\circ}$ $54^{\prime} \mathrm{S}, 12^{\circ} 28^{\prime} \mathrm{W}$ ) and the third one is from a vent site at "ABE" site on ELSC $\left(20^{\circ} 46^{\prime} \mathrm{S}, 176^{\circ} 16^{\prime} \mathrm{W}\right)$. In all these areas the seafloor positions of the venting locations were discovered using ABE.

Fig. 8 shows results from Kilo Moana vent site. This site was relatively easy. As is evident from the $3 \mathrm{D}$ image of the $M A D_{i}$, relatively strong anomalies are encountered by ABE only in the north-east corner of the survey. The proposed algorithm selects the highest intensity anomaly and triggers only one spiral. The spiral is triggered in a good location, because it causes the robot to fly over several hydrothermal vents locations that were confirmed in subsequent dives. 
A more difficult dataset is that from SMAR (see Fig. 9). This is the survey for which a basic spiral length of $150 \mathrm{~m}$ is used given that the tracklines are $1 \mathrm{~km}$ spaced. Even if the tracklines spacing is very wide, the $M A D_{i}$ show clearly three different regions of anomalies. In all three cases, a spiral is started causing the robot to fly over or very near vent fields: all three perturbations were later revealed to be related to nearby buoyant plumes. Only the three highest intensity anomalies triggered spirals: other lower Eh perturbations to the north are ignored.

Finally, a third site on the ELSC presents an even more difficult situation. From Fig. 10 it is evident that perturbations in Eh signal are present in a large part of the survey. In this survey, TSP chooses four points to trigger spirals. The two northern spirals result in trajectories that pass over or near vents. The area covered by the southern-most spiral is likely to contain some venting activity but was never confirmed: a vertical velocity anomaly has been detected in that area (Jakuba, 2007). That spiral movement suggests an interesting area to explore in next missions. The fourth spiral may be caused by the known vents' activity to the north. Finally a known venting area is "missed" by the algorithm. We consider "to miss" a venting area if the robot, during a spiral movement, does not pass at a distance shorter than $150 \mathrm{~m}$ from the area. For unknown reasons, at the locations near the missed venting area, the perturbations in Eh are very low (also perturbations in other tracers do not reveal nearby hydrothermal activity (Jakuba, 2007)).

To investigate the efficiency of the method we have analyzed the results from all 7 addressed surveys quantifying how much the triggered spirals are related to nearby hydrothermal activities.

To do this we group the spirals triggered in the different trials in three different categories:

- spirals with known nearby vents: this category includes spiral movements passing at less than $150 \mathrm{~m}$ from a vent (the diameter of a buoyant stem is $\mathrm{O}(100 \mathrm{~m}))$. So passing at that distance from a vent increases the likelihood of direct contact with a buoyant plume. The movements in this category are connected to confirmed venting activities and are the movements that we considered the best ones generated by TSP; 
- spirals with likely nearby vents: this category consists of spirals triggered in areas where off-line analysis suggests venting activity but where Phase-3 dives were never conducted to confirm that activity;

- other spirals: this category includes all other spirals. Spirals in this category are considered avoidable if the energy capacity does not permit to cover all the trajectories suggested by TSP.

In Table 2 we report the results using TSP with the available previously collected data in terms of these categories. To investigate how the adapting threshold mechanism works we report both the results with the use of adapting mechanism and with a fixed trigger threshold $\left(\eta_{\text {trigger }}=180\right)$.

The results of the table show the efficiency of TSP approach. The high percentage of spirals belonging to the first two categories together with the limited total number of triggered spirals (the average is under the suggested maximum number of spirals (5)) indicates robustness to variable environmental conditions. The suggested number of spirals was reached only in one trial. In that trial, particularly diffuse Eh perturbations triggered 5 spirals for TSP with the adaptation mechanism and 6 spirals without adaptation. The analysis of the two versions of TSP shows that using an adapting threshold is preferable. The adaptive TSP presents a higher percentage of triggers in the first two categories and, more importantly, the TSP with a fixed trigger threshold missed two more venting areas.

However, as for every method using some thresholds to make decisions, one delicate issue is how to choose the starting threshold. In a previous section we said we chose it to be 180 times the normalized $M A D_{B}$ basing on our experience from data from previous surveys. To investigate the robustness of the chosen threshold we also ran the program with two other trigger thresholds: 140 and 220 . The three thresholds (140, 180 and 220) were used as the basic trigger threshold $\left(\eta_{\text {trigger_B }}\right)$ for the adapting mechanism and as the fixed $\eta_{\text {trigger }}$ in the fixed threshold TSP version. 

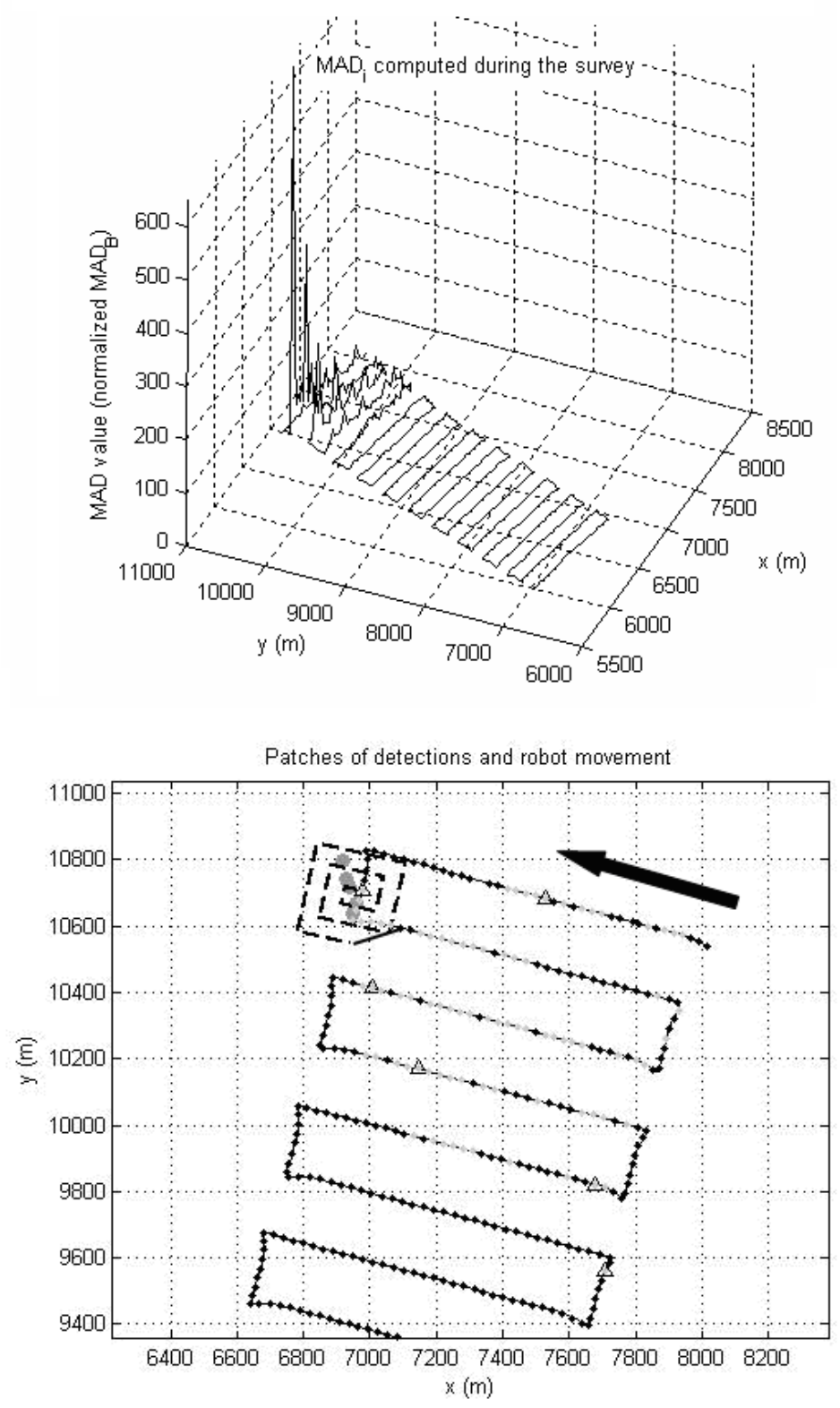

Fig. $83 \mathrm{D}$ image of created $M A D_{i}$ (top) and trajectory generated by TSP (bottom). The 3D image shows nascent hydrothermal waters intercepted to the north-east part of the survey. Robot trajectory shows one triggered spirals covering an area containing different vents (grey circles). Dots along the tracklines represent the locations of created $M A D_{i}$ : black dots are non-detections, grey ones are the detections. Triangles mark the positions of patches centroids. Finally, the black arrow shows the direction of movement of ABE. The two plots are produced using TSP with data collected in a Phase-1 dive at "Kilo Moana" site at ELSC ( $\left.20^{\circ} 3^{\prime} \mathrm{S}, 176^{\circ} 12^{\prime} \mathrm{W}\right)$ (Mid-Pacific Ridge). 

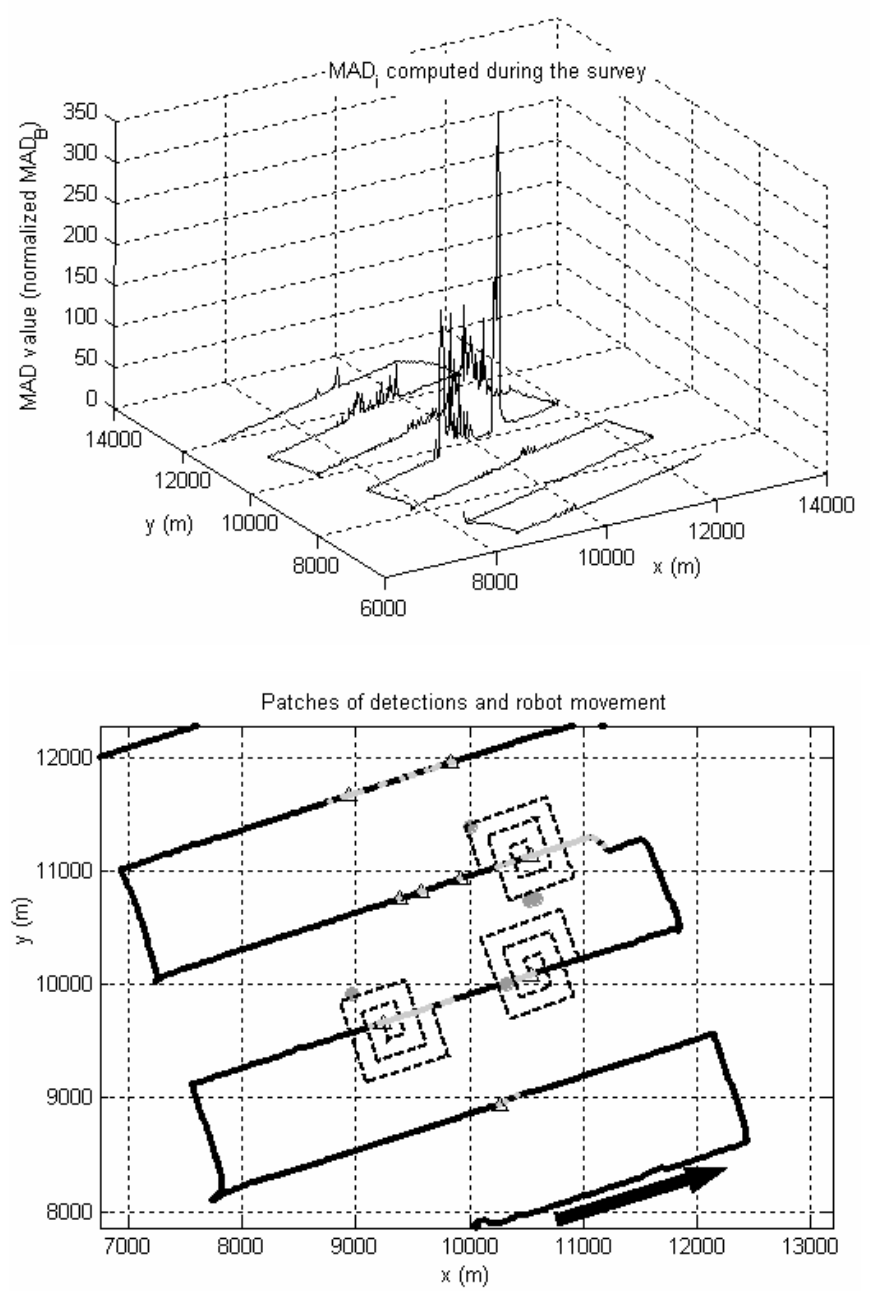

Fig. $93 \mathrm{D}$ image of created $M A D_{i}$ (top) and trajectory generated by TSP (bottom). The 3D image shows nascent hydrothermal waters intercepted in three different regions. Robot trajectory shows three triggered spirals, one for each of the three regions. The triggered movements sweep areas containing all the vents (grey circles) currently known in the site. Dots along the tracklines represent the locations of created $M A D_{i}$ : black dots are non-detections, grey ones are the detections. Triangles mark the positions of patches centroids. Finally, the black arrow shows the direction of movement of ABE. The two plots are produced using TSP with data collected in a Phase-1 dive at SMAR site (Southern Mid-Atlantic Ridge) ( $\left.4^{\circ} 54^{\prime} \mathrm{S}, 12^{\circ} 28^{\prime} \mathrm{W}\right)$. 


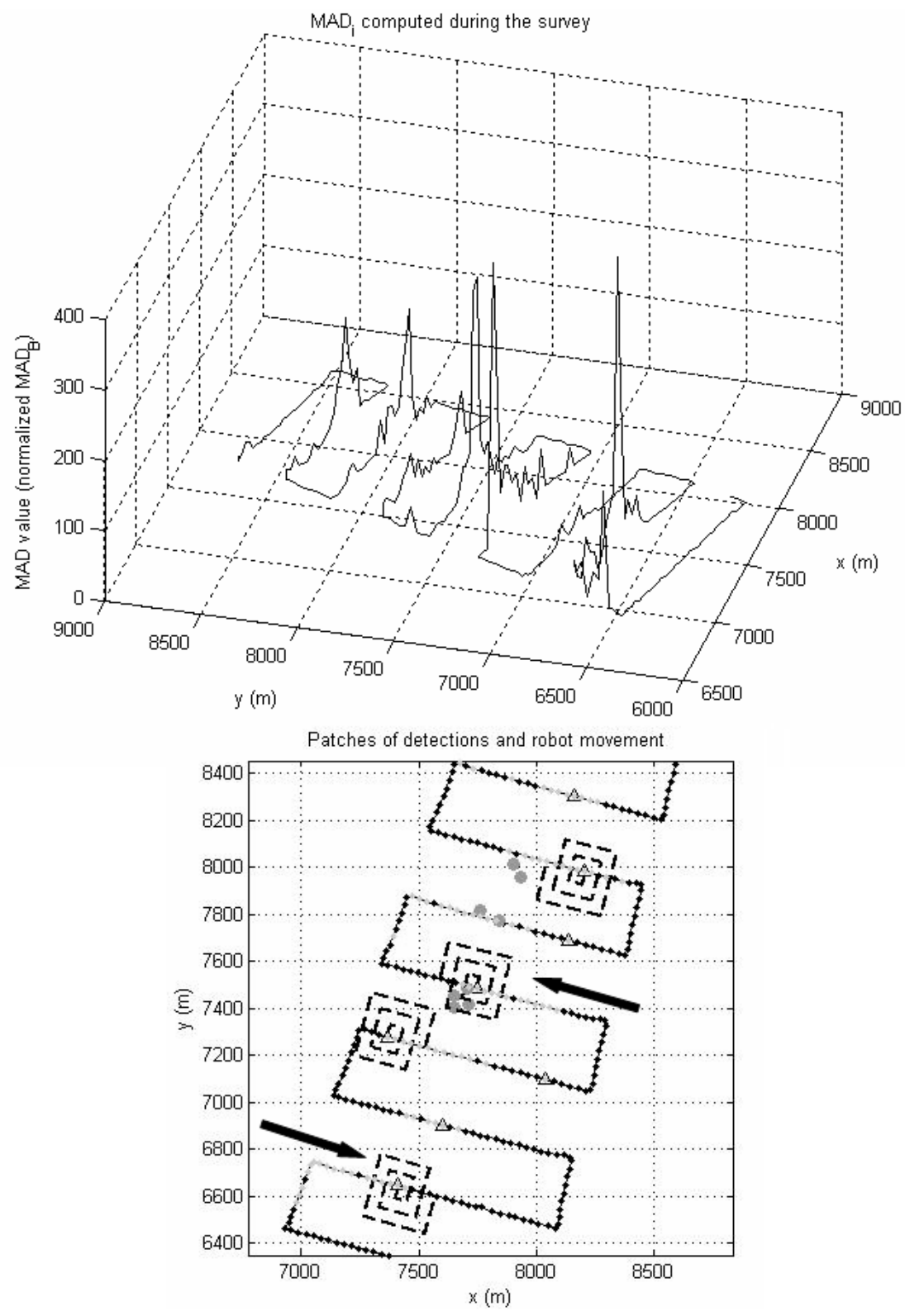

Fig. $103 \mathrm{D}$ image of created $M A D_{i}$ (top) and trajectory generated by TSP (bottom). The 3D image shows nascent hydrothermal waters intercepted in a large areas of the survey. Robot trajectory shows four triggered spirals. Three of the triggered movements are related to the activity of the vents (grey circles) currently known in the site. The most southern spiral sweeps an area that has not been explored, but presents clues of very likely nearby hydrothermal activity (a vertical velocity anomaly has been detected there). Dots along the tracklines represent the locations of created $M A D_{i}$ : black dots are non-detections, grey ones are the detections. Triangles mark the positions of patches centroids. Finally, the black arrow shows the direction of movement of ABE. The two plots are produced by TSP from data collected in a Phase- 1 dive at "ABE" site at ELSC (2046' S, 176 16' W) (Mid-Pacific Ridge).

In Table 3 the results are shown. From the results, it is clear that the adaptive mechanism gives more guarantees of robustness; for it, the resulting spirals, changing the basic trigger threshold, change only in one dataset: it is the above cited survey with diffuse perturbations. In that survey one more movement is triggered for a total of 6 spirals. 
The fixed threshold version, instead, presents more variability in the number of triggered spirals. If the threshold is lowered, the number of spirals increases producing the same results of the TSP in the adaptive form in terms of number of triggers (19), but with one more missed venting area. Even if the average number of spirals (2.71) is smaller than the suggested number, in the dataset with diffuse and strong Eh perturbations the number of triggers raises to 7 . This is a relatively large number that could not be sustainable in a real survey and, therefore, some other vents might have been missed. Otherwise, if the trigger threshold is raised, the total number of spirals for all surveys decreases to 14 causing an increased number of missed venting areas (5 representing a percentage of $38.5 \%$ on the total known venting areas). The percentage of missed known venting areas with the adaptive mechanism remains the same with all the three thresholds (15.4\%).

To summarize, even if the chosen threshold appears to be quite robust, the mechanism of threshold adaptation gives more robustness to TSP algorithm. This is due to the capability of dynamically lowering and raising the threshold based on acquired knowledge about the environment in the ongoing dive. This mechanism can be also used as a method to control the percentage of the mission assigned to spirals: a higher number of suggested spirals tends to decrease the threshold, a lower number to increase it.

Finally we show in Table 4 the total distance traversed, the total distance covered in spirals, the total estimated time of the survey and the total energy cost for just Phase-1 tracklines and for Phase-1 with spirals for the 7 datasets using TSP with threshold adapting mechanism and with a basic threshold of 180 . In all the surveys the energy cost of TSP is below ABE's endurance limit. In two cases, however, it is close to ABE's endurance limit: in the survey with $D=155$ ( $96 \%$ of available energy consumed) due to the large spirals covered caused by the wide-spaced tracklines and in the cited survey with diffuse perturbations ( 5 spirals lead to $\sim 90 \%$ of available energy consumed).

The results presented in this section show that TSP approach can be a reliable method to increase the amount of useful information about hydrothermal activity collected during Phase-1 surveys. The computed Eh anomalies proved to be well related to nearby nascent hydrothermal plumes. The created $M A D_{i}$ give also a readily interpretable map of Eh anomalies either from an intensity point of view (the intensity of $M A D_{i}$ ) or from a spatial point of view (patches delineate the Eh 
perturbed areas). Attention must be paid to cases (we have one in our datasets) in which a relatively high number of triggers occur. They may happen in particularly Eh perturbed areas. The relatively large number of triggers would not allow ABE to cover all the pre-designed tracklines. A trade-off between the portion of survey assigned to eventual triggers and the portion assigned to cover the pre-designed tracklines has to be achieved by the scientific party.

Table 2. Results using TSP with threshold adapting mechanism and with a fixed trigger threshold. TSP has been used on 7 datasets coming from previous ABE's Phase-1 dives. The shown percentages are with respect to the total number of triggered spirals and to the total number of known venting areas.

TSP with threshold TSP with fixed adapting threshold

mechanism

\begin{tabular}{ccc}
\hline $\begin{array}{c}\text { \# spirals with } \\
\text { confirmed } \\
\text { nearby vents }\end{array}$ & $11(61.1 \%)$ & $9(56.3 \%)$ \\
\hline \# spirals with & & \\
likely & & $4(25 \%)$ \\
nearby vents & $5(27.7 \%)$ & $3(18.8 \%)$ \\
\hline \# other spirals & $2(11.1 \%)$ & $4(30.8 \%)$ \\
\hline \# missed & & \\
venting areas & $2(15.4 \%)$ & total=16 \\
\hline \# triggered & & $\mu=2.29$ \\
& & $\sigma=1.67$ \\
\hline
\end{tabular}


Table 3. Number of triggered spirals and missed venting areas, using TSP with adapting mechanism and TSP with a fixed threshold. Three different basic thresholds are considered. These results are produced by TSP using 7 datasets coming from previous ABE's Phase-1. The percentages are with respect to the total number of known venting areas.

\begin{tabular}{|c|c|c|c|c|}
\hline & \multicolumn{2}{|c|}{$\begin{array}{l}\text { TSP with threshold } \\
\text { adapting mechanism }\end{array}$} & \multicolumn{2}{|c|}{$\begin{array}{l}\text { TSP with fixed } \\
\text { threshold }\end{array}$} \\
\hline & \# spirals & $\begin{array}{c}\text { \# missed } \\
\text { venting } \\
\text { areas }\end{array}$ & \# spirals & $\begin{array}{c}\text { \# missed } \\
\text { venting } \\
\text { areas }\end{array}$ \\
\hline \multirow{3}{*}{$\eta_{\text {trigger_B }}=140$} & total $=19$ & & total $=19$ & \\
\hline & $\mu=2.71$ & $2(15.4 \%)$ & $\mu=2.71$ & $3(23.1 \%)$ \\
\hline & $\sigma=1.67$ & & $\sigma=1.98$ & \\
\hline \multirow{3}{*}{$\eta_{\text {trigger_B } B}=180$} & total $=18$ & & total $=16$ & \\
\hline & $\mu=2.57$ & $2(15.4 \%)$ & $\mu=2.29$ & $4(30.8 \%)$ \\
\hline & $\sigma=1.4$ & & $\sigma=1.67$ & \\
\hline \multirow{3}{*}{$\eta_{\text {trigger } \_B}=220$} & total $=18$ & & total $=14$ & \\
\hline & $\mu=2.57$ & $2(15.4 \%)$ & $\mu=2$ & $5(38.5 \%)$ \\
\hline & $\sigma=1.4$ & & $\sigma=1.85$ & \\
\hline
\end{tabular}


Table 4. Total distance traversed (considering the triggered spirals and the additional space covered for backtracking when one cluster is closed), total distance covered during spirals, total estimated time for survey using TSP algorithm, total energy cost for just Phase-1 tracklines and for Phase-1 with spirals for the 7 considered ABE's surveys. TSP algorithm was run with threshold adapting mechanism and with a basic threshold of 180 . The energy cost is expressed in percentage with respect to an available ABE's energy sufficient for covering $50 \mathrm{~km}$ in one survey. The first three surveys are those described in detail in the text.

\begin{tabular}{|c|c|c|c|c|c|}
\hline & $\begin{array}{c}\text { Total } \\
\text { distance } \\
\text { traversed } \\
\text { (m) }\end{array}$ & $\begin{array}{l}\text { Total distance } \\
\text { in spirals (m) }\end{array}$ & $\begin{array}{c}\text { Total } \\
\text { estimated } \\
\text { time (hrs) }\end{array}$ & $\begin{array}{c}\text { Total energy } \\
\text { cost for just } \\
\text { Phase-1 } \\
\text { tracklines } \\
\text { (percentage } \\
\text { with respect } \\
\text { ABE's } \\
\text { available } \\
\text { energy) }\end{array}$ & $\begin{array}{c}\text { Total energy } \\
\text { cost Phase-1 } \\
\text { with spirals } \\
\text { (percentage } \\
\text { with respect } \\
\text { ABE's } \\
\text { available } \\
\text { energy) }\end{array}$ \\
\hline $\begin{array}{l}\text { Survey } 1 \text { (dive at } \\
\text { “Kilo Moana” site } \\
\text { at ELSC, Mid- } \\
\text { Pacific Ridge - } \\
\text { see Fig. 8) }\end{array}$ & 28810 & $\begin{array}{c}1815 \text { (1 spiral, } \\
D=55)\end{array}$ & 13.34 & $52.4 \%$ & $57.62 \%$ \\
\hline $\begin{array}{c}\text { Survey } 2 \text { (dive at } \\
\text { SMAR site, } \\
\text { Southern Mid- } \\
\text { Atlantic Ridge - } \\
\text { see Fig. 9) }\end{array}$ & 48250 & $\begin{array}{c}14850 \text { (3 spirals, } \\
D=155)\end{array}$ & 22.33 & $64.8 \%$ & $96.5 \%$ \\
\hline $\begin{array}{c}\text { Survey } 3 \text { (dive at } \\
\text { SMAR site, } \\
\text { Southern Mid- } \\
\text { Atlantic Ridge - } \\
\text { see Fig. 10) }\end{array}$ & 21480 & $\begin{array}{c}7260 \text { (4 spirals, } \\
D=55)\end{array}$ & 9.94 & $25.24 \%$ & $42.96 \%$ \\
\hline Survey 4 & 14676 & $\begin{array}{c}1815 \text { (1 spiral, } \\
D=55)\end{array}$ & 6.8 & $24.82 \%$ & $29.35 \%$ \\
\hline Survey 5 & 45124 & $\begin{array}{c}9075 \text { (5 spirals, } \\
D=55)\end{array}$ & 20.89 & $63.08 \%$ & $90.24 \%$ \\
\hline Survey 6 & 22073 & $\begin{array}{c}3630 \text { (2 spirals, } \\
D=55)\end{array}$ & 10.21 & $34.31 \%$ & $44.14 \%$ \\
\hline Survey 7 & 33890 & $\begin{array}{c}3630 \text { (2 spirals, } \\
D=55)\end{array}$ & 15.68 & $56.92 \%$ & $67.78 \%$ \\
\hline
\end{tabular}




\section{Conclusions and future works}

In this paper we address the problem of localizing hydrothermal vents using an AUV. We present a method (TSP) to improve ABE's movements during Phase-1 surveys. This phase, due to the largest altitude, appears to be the most difficult one in the three-stage nested strategy (German et al., 2008) used in recent ABE's missions. Autonomous movements, driven by acquired data, covering areas potentially containing vents, would increase the amount of useful data to localize vent fields and reduce the number of dives needed to localize the venting areas locations. TSP aims at mixing the robustness of pre-designed tracklines with the efficiency of data-driven algorithms without considering the often misleading and difficult to analyze information carried by the water current: $\mathrm{ABE}$ follows preplanned tracklines starting an explorative movement (a spiral) when some conditions on acquired data suggest nearby nascent hydrothermal water (the considered tracer was the Eh potential).

Our approach has been tested on 7 datasets collected during Phase-1 dives. It proved to work well with a percentage of $88.8 \%$ of spirals, carrying likely useful information to localize the vents, triggered in very interesting areas. The twolayered cluster method, together with the adaptive threshold mechanism (that also improves the robustness to changes in starting threshold) appears to be able to select the highest anomalies locations of a survey without requiring a complete characterization of the survey.

The number of triggered spirals was on average 2.57: it is an acceptable number taking in consideration the additional distance $\mathrm{ABE}$ has to cover during the spirals. One trial, however, resulted in 5 spirals (6 spirals with the lowest used basic threshold), a quite large number. This may happen in surveys with high intensity anomalies. They could be avoided fixing the percentage of the mission assigned to spiral movements.

We propose possible solutions that we are going to investigate in the future. A possible strategy could be giving the priority to triggered movements, even if this implies not covering all pre-designed tracklines: it could be preferable for the scientific party to cover a smaller area, but to be reasonably sure that the locations likely to contain hydrothermal vents in the covered area have been investigated. An alternative strategy could be that of fixing a maximum percentage of the 
survey time assigned to spirals. These are two possible strategies: the first one sacrifices the exploration of the pre-planned area to investigate with spirals interesting areas, the second one sacrifices the exploration of eventual interesting areas but guarantees the coverage of preplanned tracklines. A more complex solution could be not using pre-designed spirals in length. After a spiral is triggered, if during the movement no high Eh perturbations are encountered, ABE could come back to move on the tracklines aborting the actual spiral. In this way $\mathrm{ABE}$ would have the possibility to trigger on all interesting areas, but continuing the movement only if relatively high Eh perturbations were found. In addition, a methodology as in (Ferri et al., 2009) with propagated spirals (a new spiral is triggered during a previous one if high anomalies are encountered) could help ABE moving towards interesting areas. This approach would minimize the wasted time covering areas not presenting interesting clues about hydrothermal activities, not losing the possibility of triggering at potentially interesting locations. In the future, TSP algorithm will be implemented on ABE and used in Phase-1 surveys for hydrothermal vents prospecting. The acquired data will also be useful to design the above described strategy.

Finally, TSP can be also used together with mapping techniques. The data carried by spirals triggered at "right" locations can be used by the mapping method proposed in (Jakuba, 2007) to produce probabilistic maps of a vent sites: these maps would be more accurate with respect to those produced by passing only on the tracklines, because more direct interceptions of buoyant plumes would be incorporated in the measurements to update the maps.

\section{REFERENCES}

Baker, E.T., German, C.R., Elderfield, H. (1995). Hydrothermal plumes over spreading-center axes: global distributions and geological inferences. In American Geophysical Union, Geophysical Monograph 91, Seafloor hydrothermal systems: physical, chemical, biological, and geological interaction.

Burian, E., Yoerger, D., Bradley, A., Singh, H. (1996). Gradient search with autonomous underwater vehicles using scalar measurements. In Proceedings AUV '96, Monterey, CA, USA, 86-98.

Corliss, J., Dymond, J., Gordon, L.I., Edmond, J.M, Von Herzen, R.P, Ballard, R.D., Green, K, Williams D, Bainbridge A, Crane K., van Andel TH (1979). Submarine thermal springs on the Galapagos Rift. Science, 203, 1073-1083.

Ferri, G., Caselli, E., Mattoli, V., Mondini, A., Mazzolai, B., Dario, P. (2009). SPIRAL: a Novel Biologically-Inspired Algorithm for Gas/Odor Source Localization in an Indoor Environment with no Strong Airflow. Robotics and Autonomous Systems, Volume 57, Issue 4, 30 April 2009, 393-402.

Fisher, C.R. (1995). Toward an appreciation of hydrothermal-vent animals: their environment, physiological ecology, and tissue stable isotope values. In American Geophysical Union, Geophysical Monograph 91, 
Seafloor hydrothermal systems: physical, chemical, biological, and geological interaction.

German, C.R., Connelly, D.P., Prien, R., Yoerger, D., Jakuba, M., Bradley, A., Shank, T., Nakamura, K., Langmuir, C., Parsons, L.M. (2005). New techniques for hydrothermal plume investigation by AUV. Geophysical Research Abstracts, volume 7, Vienna, Austria, 2005. European Geosciences Union.

German, C., Yoerger, D., Jakuba, M., Shank, T., Langmuir, C., Nakamura, K. (2008). Hydrothermal exploration with the Autonomous Benthic Explorer. Deep Sea Research, Volume I, 55, 203-219.

Helfrich, K. R., and Speer, K. G. (1995) Oceanic hydrothermal circulation: mesoscale and basin-scale flow. In American Geophysical Union, Geophysical Monograph 91, Seafloor hydrothermal systems: physical, chemical, biological, and geological interactions.

Huber, P. (1981). Robust statistics, New York: Wiley.

Jakuba, M., Yoerger, D., Bradley, A., German, C., Langmuir, C., Shank, T. (2005). Multiscale, multimodal AUV surveys for hydrothermal vent localization. In Proceedings of the Fourteenth International Symposium on Unmanned Untethered Submersible Technology (UUST05), Durham, NH.

Jakuba, M.V. (2007). Stochastic mapping for chemical plume source localization with application to autonomous hydrothermal vent discovery. PhD Thesis, WHOI-MIT.

Kadko, D., Baross, J., Alt, J. (1995). The magnitude and global implications of hydrothermal flux. In American Geophysical Union, Geophysical Monograph 91, Seafloor hydrothermal systems: physical, chemical, biological, and geological interactions.

Langmuir, C.H, German, C., Michael, P., Yoerger, D.R., Fornari, D.J, Shank, T.M., Asimow, P,D., Edmonds, H.N., Lau2TEAM (2004). Hydrothermal prospecting and petrological sampling in the Lau Basin: background data for the integrated study site. Eos. Trans. AGU, volume 85. Fall Meet. Suppl., Abstract B13A-0189.

Lavelle, J. W. (1997). Buoyancy-driven plumes in rotating, stratified cross flows: Plume dependence on rotation, turbulent mixing, and cross-flow strength. Journal of Geophysical Research, 102 (C2), 34053420

Li, W., Farrell, J.A., Pang, S., Arrieta, R. (2006). Moth-inspired chemical plume tracing on an autonomous underwater vehicle. IEEE Transactions on Robotics, Vol.22, No. 2, $292-307$.

Lupton, J.E. (1995). Hydrothermal plumes: near and far field. In American Geophysical Union, Geophysical Monograph 91, Seafloor hydrothermal systems: physical, chemical, biological, and geological interaction.

McDuff, R.E. (1995). Physical dynamics of deep-sea hydrothermal plumes. In: American Geophysical Union, Geophysical Monograph 91, Seafloor hydrothermal systems: physical, chemical, biological, and geological interactions.

Menold, P.H., Pearson, R.K., Allgower, F. (1999). Online outlier detection and removal. In Proceedings of the 7th Mediterranean Conference on Control and Automation (MED99), Haifa, Israel.

Nakamura, K., Veirs, S., Sarason, C.P., McDuff, R.E., Stahr, F., Yoerger, D.R, Bradley, A.M. (2000). Chemical signals in rising buoyant plumes and tidally oscillating plumes at the Main Endeavour vent field, Juan de Fuca Ridge. Eos. Trans. AGU, volume 81, 2000. Fall Meet. Suppl., Abstract OS52I-05.

Thompson, R.E., Milhàly, S.F., Rabinovich, A.B., McDuff, R.E., Veirs, S.R., Stahr, F.R. (2003). Constrained circulation at Endeavour ridge facilitates colonization by vent larvae. Nature, 424(31), 545-549.

Turner, J.S. (1986). Turbulent entrainment: the development of the entrainment assumption, and its application to geophysical flows. Journal of Fluid Mechanics, 173, 431-471.

Veirs S (2003). Submarine volcanic heat flux and hydrography: observations and models of the Main Endeavour vent field in the Northeast Pacific. PhD Thesis, University of Washington.

Yoerger, D.R., Bradley, A.M., Warren, B.B. (1991). The Autonomous Benthic Explorer (ABE): an AUV optimized for deep seafloor studies. In Proceedings of the Seventh International Symposium on 
Unmanned Untethered Submersible Technology (UUST91), Durham, NH, 60-70.

Yoerger, D.R., Jakuba, M.V., Bradley, A.M., Bingham, B. (2007). Techniques for deep sea near bottom survey using an autonomous underwater robot. International Journal of Robotics Research. Volume 26, Issue 1 (January 2007), 41-54.

Zierenberg R.A., Adams M.W.W., Arp A.J. (2000). Life in extreme environments: hydrothermal vents. Proceedings of the National Academy of Sciences of the United States of America, vol. 97, no. 24, 12961-12962.

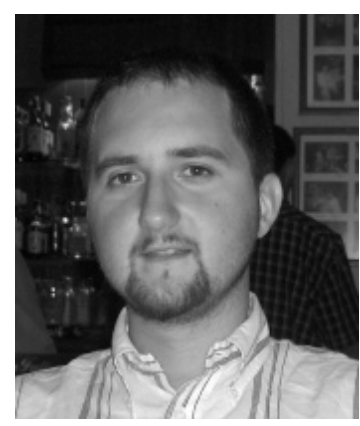

Gabriele Ferri received the Laurea degree (M.S.) in Computer Engineering (with Honors) from the University of Pisa, Italy, in 2003. From 2003 to 2005, he worked as a Software/System Consultant Engineer for WASS company (a Finmeccanica group company) in Livorno, Italy, working on the design of a new System of Control and Guidance for a light-weighted torpedo. He earned a Ph.D. in Biorobotic Science and Engineering jointly from IMT Advanced Studies in Lucca, Italy and Scuola Superiore S. Anna, Pisa, Italy in 2008. He was a visiting researcher for one year at Woods Hole Oceanographic Institution, Woods Hole, Massachusetts, USA. Currently, he is a Postdoctoral Investigator at CRIM Lab, Scuola Superiore Sant'Anna. His research interests include robotic systems for environmental monitoring/exploration, control theory and robot navigation issues.

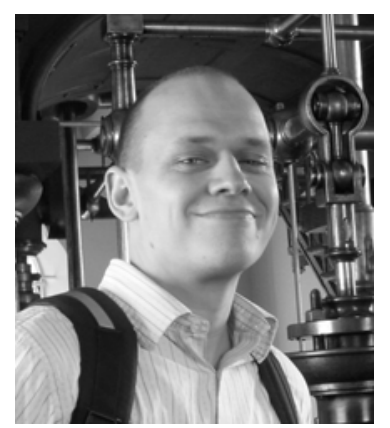

Michael V. Jakuba is presently a postdoctoral fellow at the Australian Centre for Field Robotics at the University of Sydney. His research interests revolve around the development of underwater robots to facilitate ocean science, particularly through high resolution and long term autonomous benthic mapping. Dr. Jakuba earned his doctorate from the MIT/WHOI Joint Program in Applied Ocean Physics and Engineering in 2007. His thesis work developed novel mapping algorithms for the autonomous localization of hydrothermal venting.

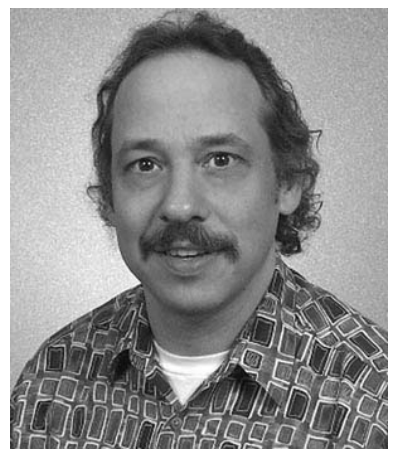

Dana R. Yoerger received the S.B., S.M., and Ph.D. degrees in Mechanical Engineering from the Massachusetts Institute of Technology. He is currently a Senior Scientist at the Woods Hole Oceanographic Institution, where he designs and implements robotic control systems for telerobotic and autonomous underwater vehicles. He supervises the thesis research of students enrolled in the WHOI/MIT joint program in Oceanographic Engineering, in the areas of control, robotics, and design. Dr. Yoerger has gone to sea on over 50 oceanographic expeditions, including the 1985 Titanic discovery cruise. He was a member of the team that developed the telerobotic control system for the Jason ROV and has served as Jason navigator on many cruises. For operations of the fully autonomous ABE vehicle, his responsibilities include science liaison, control system design and implementation, mission programming and testing, and generation of data products. He has also participated in a number of education and outreach programs featuring deep submergence technology and seafloor exploration. These include five Jason Project expeditions and two REVEL programs. Dr. Yoerger is a member ofWHOI's Deep Submergence Laboratory (DSL) which was founded by Dr. Robert Ballard. DSL features a unique team of engineers, technicians, administrators, and students who perform engineering 
research and development, and also conduct extensive oceanographic field work under the auspices of WHOI's National Deep Submergence Facility. He also works with engineers from WHOI's Applied Engineering Laboratory and the Alvin Group. 